\title{
Magnetic-field modulation of a two-electrode direct-current plasma
}

\author{
David Slinkman, Fred Braid and Richard Sacks* \\ Department of Chemistry, University of Michigan, Ann Arbor, MI 48109, U.S.A.
}

(Received 18 April 1990; accepted 19 June 1990)

\begin{abstract}
The magnetic field produced by a pair of electromagnets is used to alter the shape and location of the current channel in a two-electrode direct-current plasma (DCP) designed for solution aerosol analysis. The magnetic field $\mathbf{B}$ is oriented normal to the electric field $\mathbf{E}$ in the plasma. This results in an $\mathbf{E}$-cross-B $(E \times B)$ drift motion of plasma electrons. Experiments are described using both $\mathrm{cw}$ and oscillating magnetic fields. With continuous wave (cw) fields of $10-20 \mathrm{G}$, the current channel can be shaped to improve sample aerosol penetration. Oscillating fields in the frequency range $25-500 \mathrm{~Hz}$ cause the current channel to oscillate. At the higher frequencies, the current channel loses its discrete character and the plasma becomes more diffuse.
\end{abstract}

\section{INTRODUCTION}

THE USE of external magnetic fields to alter and control the analytical properties of plasma devices is attracting considerable attention. Magnetic fields of less than $1 \mathrm{kG}$ have been used with several configurations of atmospheric-pressure arc [1-13] and low-pressure glow [14-19] discharge plasmas. At pressures above a few Torr, it is most useful to combine the magnetic field (B) with the electric field (E) in the plasma to produce a drift motion of plasma electrons which is normal to the plane containing the magnetic field and the electric field vectors. This $\mathbf{E}$-cross-B $(\mathbf{E} \times \mathbf{B})$ drift motion can be significant at relatively low magnetic field strengths where, at atmospheric pressure, the magnetic field alone would have little effect on the macroscopic properties of the plasma.

Sample introduction is a significant problem with atmospheric-pressure arc devices. The arc current channel is very localized, and the temperature gradients near the current channel are very large. This results in a thermal barrier to aerosol penetration [20-23]. The forces which repel the sample droplets or desolvated particles have been discussed [24]. Poor sample penetration may limit the power of detection with some arc devices.

Several plasma jet devices have been used to improve sample-plasma interaction [21,25-32]. These devices rely on special electrode configurations and tailored gas flows to force the sample aerosol into the hottest regions of the plasma. Turbulent gas flows in some of these devices may result in a relatively noisy signal, and high continuum background intensities may degrade the power of detection.

The use of magnetic fields to improve sample-plasma interaction with dc arc plasmas has been recently reported [1,2]. SLINKMAN and SACKS [4,5] used permanent ceramic magnets to induce a rotation of the current channel in an arc device. The system uses a hollow cylindrical graphite anode and a co-axial $\mathrm{W} / \mathrm{Th}$ cathode. The arc rotates at a frequency in the $1-3 \mathrm{kHz}$ range. The result is a diffuse plasma sheet which is easily penetrated by the sample aerosol. TRIVEDI et al. $[1,2]$ used small electromagnets with a two-electrode Ar direct-current plasma (DCP) of conventional design to cause a displacement of the current channel. By the use of an oscillating magnetic field, the channel location could be made to oscillate in a vertical plane. Improved aerosol penetration was noted. These studies were limited to an oscillation frequency of $60 \mathrm{~Hz}$ (mains frequency in U.S.A.).

In the present study, an improved magnetically modulated two-electrode DCP is described. Magnetic field frequency can be varied continuously over the range

\footnotetext{
* Corresponding author.
} 
0-500 $\mathrm{Hz}$. In addition, the plasma (electrode) angle is adjustable. This has resulted in much quieter operation with greater current-channel definition. The system is combined with a glass-frit nebulizer [33,34]. The monodisperse droplet size from the nebulizer is useful for studies of plasma structure and sample penetration. Data are presented on the effects of magnetic field strength and frequency on plasma structure and sample penetration.

\section{Apparatus and Experiment Design}

\subsection{Arc and magnet design}

Figure la shows a side section view of the device. The DCP is oriented between the poles of a pair of electromagnets $C$. One of the magnet cores has a hole through it for viewing radiation. The DCP is of conventional design using W/Th rods as electrodes and cylindrical ceramic sleeves surrounding the electrodes to deliver the Ar plasma gas. Construction details of the DCP and the magnets are found in Table 1 . Figure $1 b$ shows an end-on view of the DCP and the orientation of the electric field vector $\mathbf{E}$, the magnetic field vector $\mathbf{B}$ and the resulting $\mathbf{E} \times \mathbf{B}$ drift vector. The electric field nearly follows the locus of the current channel; while the magnetic field is nearly linear and normal to the plane of the figure. The $\mathbf{E} \times \mathbf{B}$ drift is always in the plane of the current channel and results in a breathing-like motion of the channel. The interelectrode angle is adjustable. An angle of about $95^{\circ}$ results in high plasma stability, even with considerable magnetically-induced distortion of the current channel.

An adjustable 40-V, 1-A dc power supply (Universal Electronics Model L3501) was used to generate the magnetic field for continuous wave (cw) operation. An audio-frequency function generator was used with a low-frequency power amplifier (fabricated in house) to generate oscillating magnetic fields. Because of the very low coil impedance of the magnets used here, it was difficult to obtain sufficient field strength at frequencies greater than about $500 \mathrm{~Hz}$. The peak field amplitude also was adjustable, and for each frequency value investigated, the amplitude of the function generator signal was adjusted to obtain the same value of peak coil current and thus peak magnetic field strength.

Sample aerosols of test analytes were generated with a glass-frit nebulizer. The system has been described [5]. The frit has a mean pore diameter of about $4-5.5 \mu \mathrm{m}$. The wet aerosol as

(a)

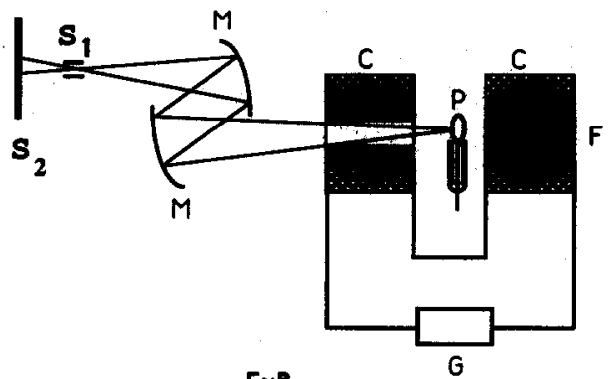

(b)

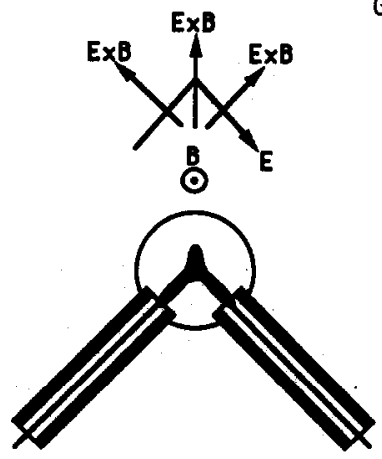

Fig. 1. (a) Side-section view of the two-electrode DCP in the pole gap of a pair of electromagnets, and (b), end-section view showing the orientation of the electrodes, the electric field, the magnetic field and the $\mathbf{E} \times \mathbf{B}$ drift motion. P, plasma; $C$, magnet coils; F, magnet cores; $G$, adjustable frequency/amplitude power supply; $M$, mirrors; $S$, slits; $E$, electric field vector; B, magnetic field vector; $\mathbf{E} \times \mathbf{B}$, electron drift vector. 
Table 1. Gas-stabilized arc and electromagnet properties

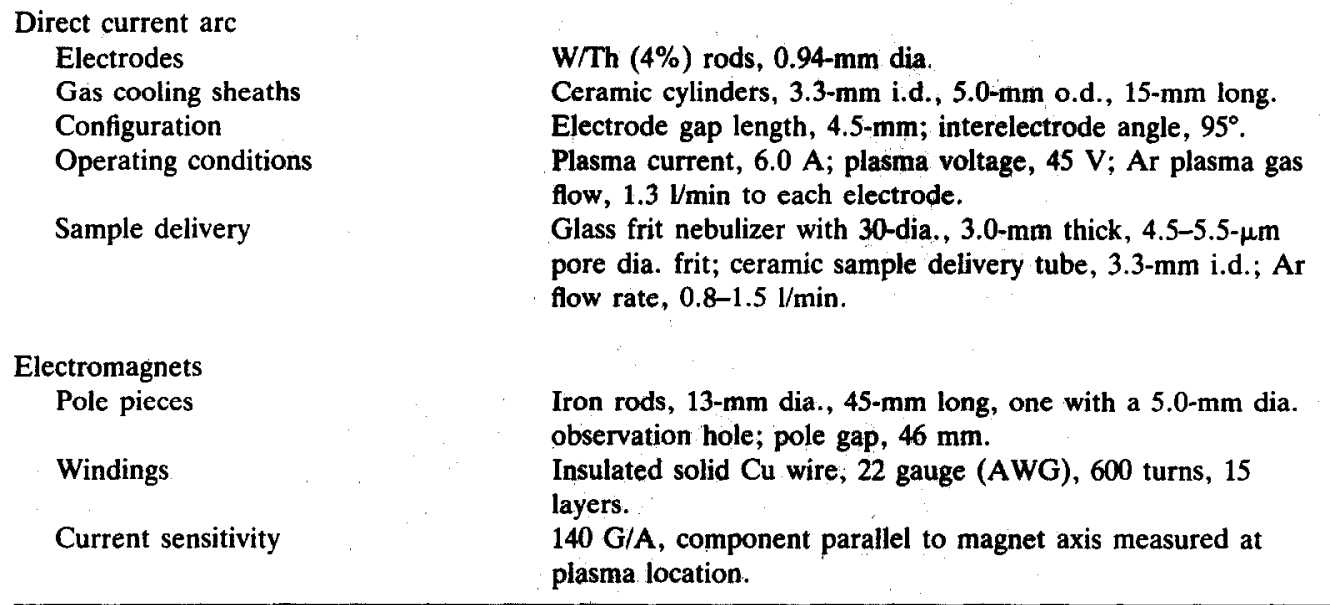

desolvated in a heated chamber, and most of the water was removed with a condenser. The dry sample aerosol was introduced to the plasma through a $3.0-\mathrm{mm}$ i.d. ceramic tube. The top of the tube was located $5 \mathrm{~mm}$ below the interelectrode axis. Sample flow rate typically was about $0.09 \mathrm{ml} / \mathrm{min}$, , and the Ar flow in the nebulizer was $2.5 \mathrm{l} / \mathrm{min}$.

\subsection{Optical and electrical monitoring}

An astigmatic image transfer system was used to obtain spatially resolved radiation measurements. A pair of $50-\mathrm{mm}$ dia., $500-\mathrm{mm}$ focal length spherical mirrors in an over-andunder configuration (see $M$ in Fig.1) was used with a magnification of 2.0. A horizontal slit $S_{1}$ placed in front of the vertical spectrometer slit $S_{2}$ was used to obtain vertical spatial resolution of about $0.1 \mathrm{~mm}$ in the plane of the current channel.

All spectra were obtained with a $1.0-\mathrm{m}$ Czerny-Turner spectrometer using a $1200-\mathrm{line} / \mathrm{mm}$ grating with a first-order blaze of $300 \mathrm{~nm}$. The instrument was used with $60-\mu \mathrm{m}$ slits giving a spectral bandwidth of about $0.05 \mathrm{~nm}$. Radiation measurements were made with a 1P28 photomultiplier tube. Bias voltage, load resistance and circuit time constant were chosen as needed. Magnetic field measurements for $\mathrm{cw}$ magnetic fields were made with a Hall-effect Gaussmeter (Central Scientific, Model 100). Field strength was measured as a function of coil current, and this calibration was used to determine peak field strength for oscillating magnetic fields. The oscillating coil current was monitored either by an integrating Rogowski coil (Pearson Electronics, Model 1025) or by the voltage drop across a $0.1 \Omega$ resistor in series with the coils.

For experiments using oscillating magnetic fields, Intensity vs time data were recorded on a Nicolet Model 2090A digital storage oscilloscope. The oscilloscope was triggered from the function generator used to generate the oscillating magnetic field waveform. The field waveform was displayed simultaneously on the oscilloscope. Background correction for Ar and W (plasma species) intensity profiles used intensity vs time profiles obtained in a line-free region near the line of interest. Phase jitter between the line and background waveforms was insignificant, and a point-by-point subtraction was used for background correction.

\section{Results AND Discussion}

\subsection{Spatial emission profiles with $\mathrm{cw}$ magnetic fields}

Figure 2 shows photographs of the arc with no magnetic field (a), with a 7.0-G magnetic field (b) and with a 15-G magnetic field (c). The arc current was $6.0 \mathrm{~A}$, and the Ar cooling gas flow rate was $1.3 \mathrm{l} / \mathrm{min}$ to each electrode. Note that the electrode axis was bent to form a $95^{\circ}$ included angle.

In the absence of a magnetic field, a well-defined, highly luminous plasma current channel is seen to bridge the electrode gap. The inverted " $V$ " shape of the current channel is the result of a number of factors including convective distortion and the 

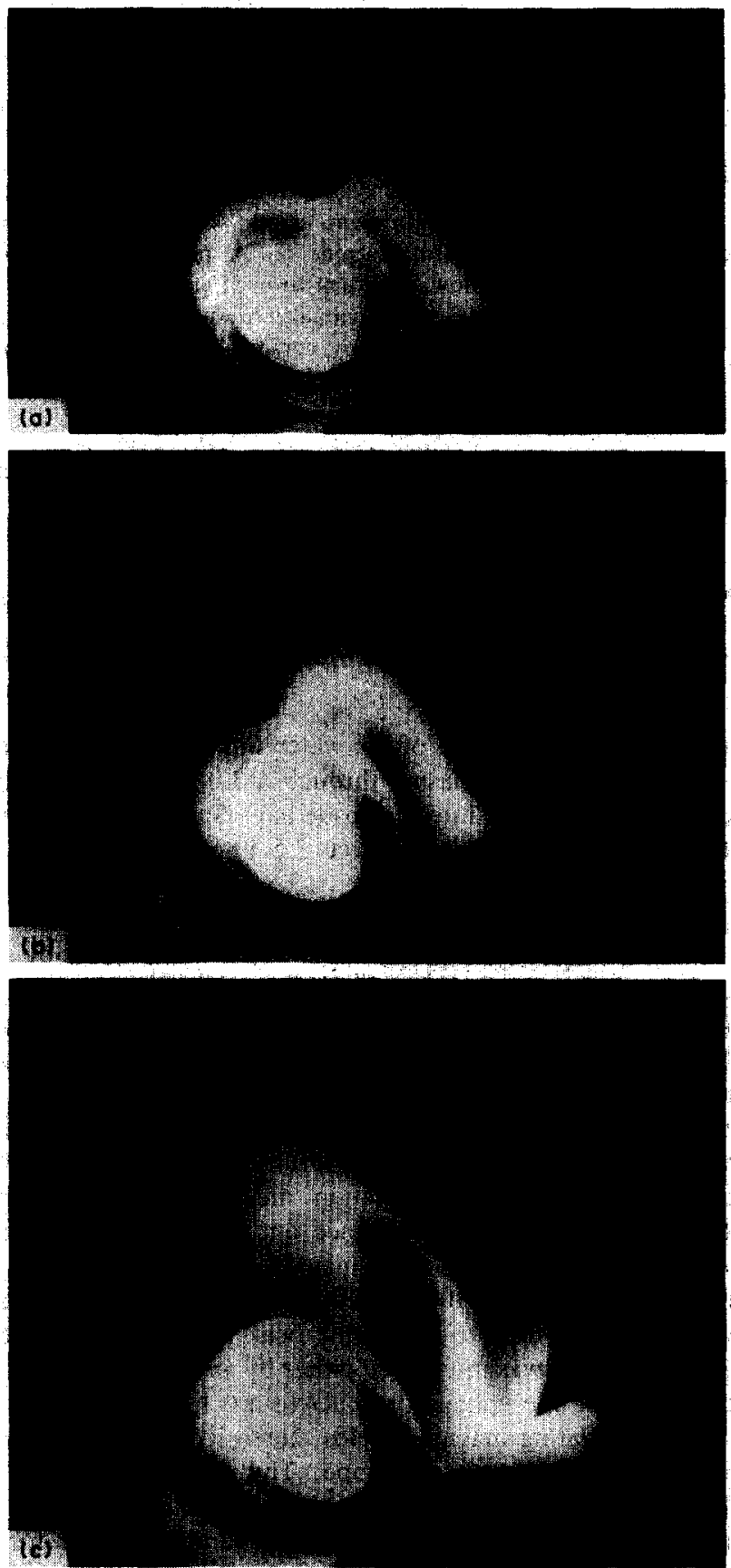

Fig. 2. Photographs of the arc plasma with no magnetic field (a), with a 7.0-G field (b) and with a 15-G field (c). In all cases, the arc current was $6.0 \mathrm{~A}$, and the Ar cooling gas flow rate was $1.3 \mathrm{l} / \mathrm{min}$ for each electrode. The electrode gap was $4.5 \mathrm{~mm}$.

flow pattern of the Ar cooling gas. Note that the more diffuse mantel above the current channel is quite transparent and only slightly luminous.

When the 7.0-G magnetic field is applied, the current channel is slightly deflected in the upward direction. Using the top of the ceramic cooling sheath as a reference point, the apex of the current channel appears to be deflected about $1 \mathrm{~mm}$ from its field-free location. With the field present, the mantel also appears brighter and large.

In the presence of the 15-G field, the appearance of the plasma changes dramatically. The apex of the current channel is driven upward about $3 \mathrm{~mm}$ from its field-free position, and in this region, the channel appears significantly thinner than for the other 
cases. The current channel has a more-or-less horseshoe shape with the stronger field. Note that near the apex of the channel, the plasma electric field vector is nearly horizontal, and the $\mathbf{E} \times \mathbf{B}$ drift motion is nearly vertical. At other points along the current channel, the electric field has a significant vertical component, and the drift motion has an outward as well as an upward component. The mantel also changes significantly with the stronger field, becoming larger and more luminous.

Figure 3 shows spatially resolved emission data for the Ar I 415.9-nm line (dotted lines) and the W I 400.8-nm line (solid lines) with no magnetic field (A), with a 7.0$G$ field (B) and with a 15-G field (C). Note that Ar and $W$ are the two principal background species in the plasma. In all cases, the arc current was $6.0 \mathrm{~A}$. For these data, a vertical reference point was chosen just below the current channel, and intensity measurements were made at several vertical displacements for the reference point. The same reference point was used for all plots in Fig. 3 and in all subsequent figures in this section.

In the absence of the magnetic field (plots A), the peak Ar line intensity occurs at a vertical position of $1.3 \mathrm{~mm}$. Note that the Ar radiation is very localized in a region with a vertical extent of only about $2 \mathrm{~mm}$. For $\mathrm{W}$, the intensity is relatively independent of vertical position in the range $0.5-2.5 \mathrm{~mm}$. In the presence of a 7.0-G magnetic field (plots B), the region of greatest Ar radiation is shifted to about $1.8 \mathrm{~mm}$, but the peak intensity is only about $10 \%$ greater than without the magnetic field. The vertical position of peak $\mathrm{W}$ intensity with the $7.0-\mathrm{G}$ field also is shifted to larger values; however, the peak intensity is about a factor of two greater than without the field. Also note that the intensity gradient in the region just under the current channel $(0-1.5 \mathrm{~mm})$ is significantly greater with the field present. These trends all continue when the field strength is increased to $15 \mathrm{G}$ (plots $\mathrm{C}$ ).

The Ar I plots in Fig. 3 as well as the photographs in Fig. 2 suggest that the $\mathrm{cw}$ magnetic field causes a vertical displacement of the arc current channel, and may significantly alter the shape or macroscopic structure of the channel. Note that the field strengths used here are relatively small. There is indication in Fig. 3 that the current channel becomes somewhat more localized in the stronger field. This is consistent with the plasma photographs in Fig. 2 . The significantly greater $W$ intensities with the field present may be the result of greater penetration of $W$ vapor into the core of the plasma or increased residence time of $W$ in the observation zone. The nature of the $W$ species entering the plasma is unclear as are the processes which transport them from the electrodes into the observation region between the electrodes.

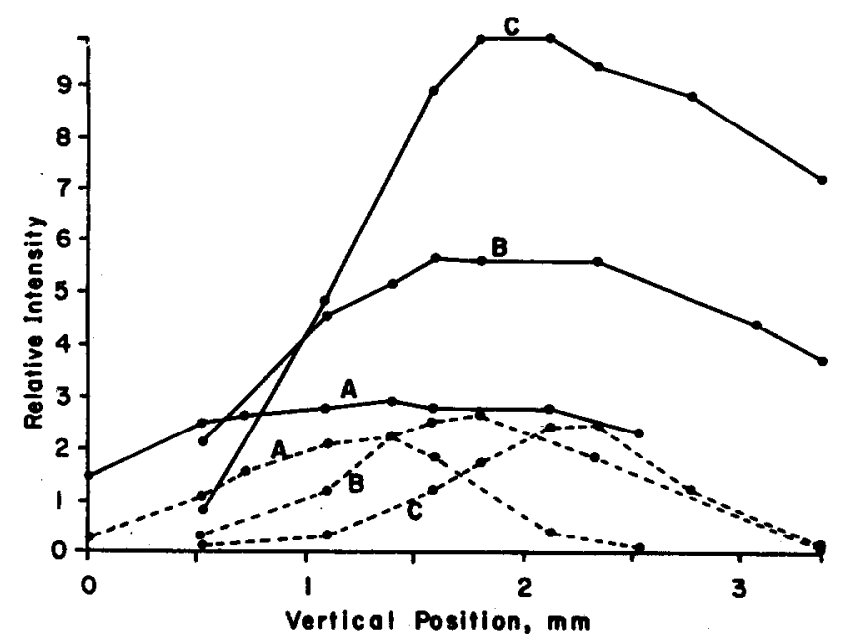

Fig. 3. Spatially-resolved emission intensities for the W I 400.8-nm line (solid-line plots) and the $\mathrm{Ar}$ I 415.9-nm line (dotted-line plots) for the cases with no magnet field (A), with a 7.0$G$ field $(B)$ and with a $14-G$ field $(C)$. For all cases, the vertical reference point was located just below the current channel for the field-free case. 
Figure 4 shows similar plots for the Mn I 403.1-nm line (solid lines) and the continuum background radiation at the analysis wavelength (dotted lines). The $\mathrm{Mn}$ was supplied as a dry aerosol from the glass frit nebulizer. A $500 \mu \mathrm{g} / \mathrm{ml}$ solution of $\mathrm{MnCl}_{2}$ was introduced into the nebulizer. The features of the continuum background plots are quite similar to the plots for the Ar I line. However, for each value of magnetic field strength, the peak background intensity occurs at a vertical position about $0.7 \mathrm{~mm}$ lower than the peak $\mathrm{Ar}$ intensity. The $\mathrm{Mn}$ plots show intensity enhancements with the magnetic field which are similar in magnitude to those observed for the $\mathrm{W}$ radiation in Fig. 3. However, the peak intensity values for Mn occur at significantly lower vertical positions for corresponding field strengths. Steeper intensity gradients at the lower vertical positions also are observed with the magnetic field present.

Figure 5 shows similar plots for the $\mathrm{Cu} \mathrm{I}$ line at $324.7 \mathrm{~nm}$ (dotted lines) and the $\mathrm{Mg}$ II line at $279.5 \mathrm{~nm}$ (solid lines). The $\mathrm{Cu}$ and $\mathrm{Mg}$ were introduced as dry aerosols from $500 \mu \mathrm{g} / \mathrm{ml}$ solutions. The $\mathrm{Cu}$ plots are quite similar to the $\mathrm{W}$ plots in Fig. 3 . The $\mathrm{Mg}$ plots show sharper intensity maxima, particularly in the presence of the magnetic field. The rapid decrease in intensity at the higher vertical positions with the ion line suggest that significant analyte ionization occurs only in a relatively restricted region near the current channel. The enhancements of peak intensity for both $\mathrm{Cu}$ and $\mathrm{Mg}$ with the magnetic field present are comparable to the values for $\mathrm{Mn}$ and $\mathrm{W}$.

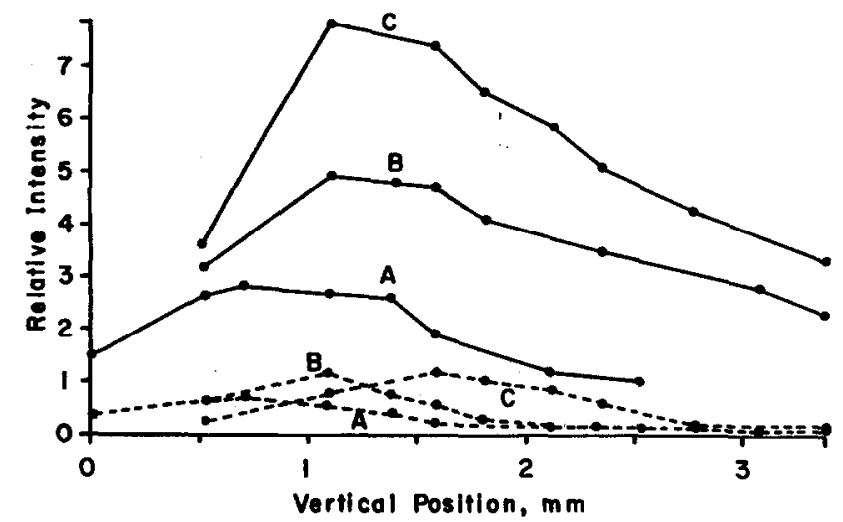

Fig. 4. Spatially-resolved emission intensities for the Mn 403.1-nm I line (solid-line plots) and continuum background (dotted-line plots) for the cases with no magnet field (A), with a 7.0$\mathrm{G}$ field (B) and with a $14-\mathrm{G}$ field (C). All conditions are the same as for Fig. 3.

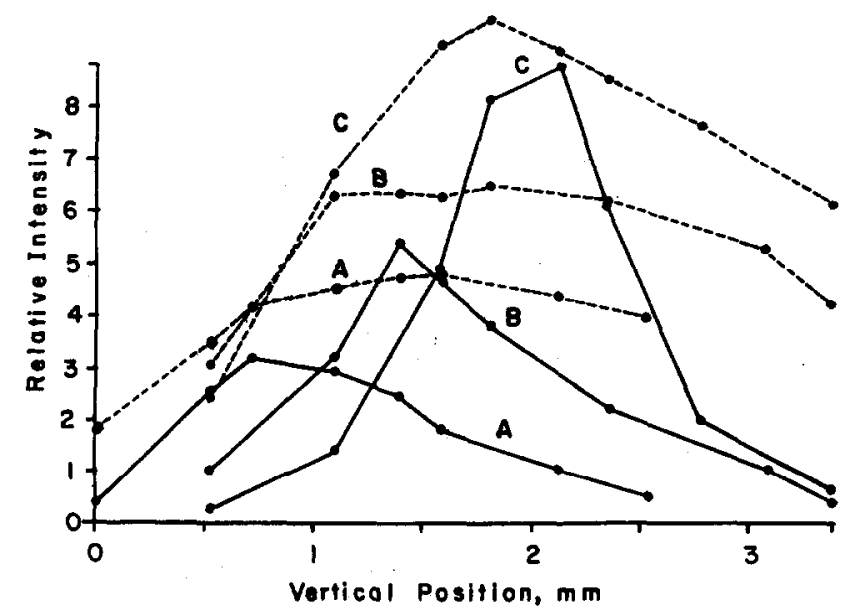

Fig. 5. Spatially-resolved emission intensities for the $\mathrm{Cu}$ I 324.7-nm line (dotted-line plots) and the $\mathrm{Mg}$ II 279.5-nm line (solid-line plots) for the cases with no magnet field (A), with a

7.0-G field (B) and with a $14-\mathrm{G}$ field (C). All conditions are the same as for Fig. 3. 
The plots in Figs 3-5 suggest that the changes in shape of the plasma current channel induced by the magnetic field increase either sample aerosol penetration or residence time. The relative invariance of peak intensities for both the Ar line and the continuum background with respect to the magnetic field strength suggest that plasma properties other than the shape and location of the current channel are not significantly changed by the magnetic field. The data suggest that stronger magnetic fields result in greater analysis line-to-background ratios. However, for the gas flow rates and plasma current used in this study, magnetic fields much stronger than $30 \mathrm{G}$ result in lower plasma stability. The $\mathbf{E} \times \mathbf{B}$ drift motion of the plasma electrons in the presence of the magnetic field appears to cause a stretching of the current channel. This is inevitable since the channel is anchored at the electrode tips at both ends. The instability observed with stronger fields is probably the result of stresses caused by excessive stretching of the current channel from its field-free equilibrium position.

\subsection{Temporal emission profiles with oscillating magnetic fields}

In the presence of an oscillating magnetic field, the current channel undergoes a periodic displacement from its field-free equilibrium position. For the studies reported here, a single, fixed observation window was located just above the current channel for the field-free case. Figure 6 shows the waveforms for an Ar I line (a), an Ar II line (b), a W I line (c) and the continuum background (d). The magnetic field is shown in waveform (e). The field frequency was about $25 \mathrm{~Hz}$, and the peak amplitude was about $25 \mathrm{G}$.

The Ar I line and the continuum background waveforms show qualitatively similar behavior with nearly equally spaced local maxima occurring near the time of each zero crossing of the magnetic field waveform. Local minima occur at both positive and negative peaks of the field waveform. There is some asymmetry with more pronounced

(a)

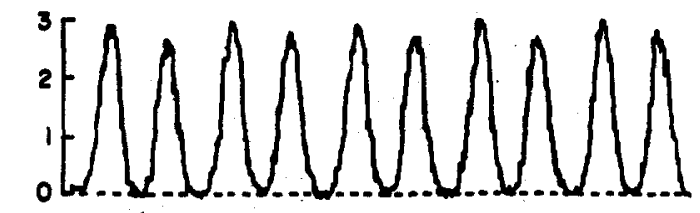

(b)

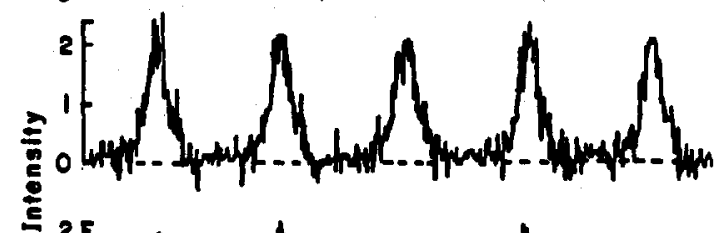

(c)

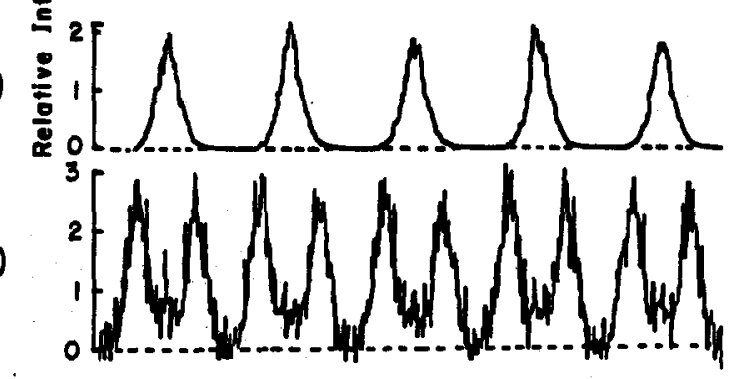

(e)

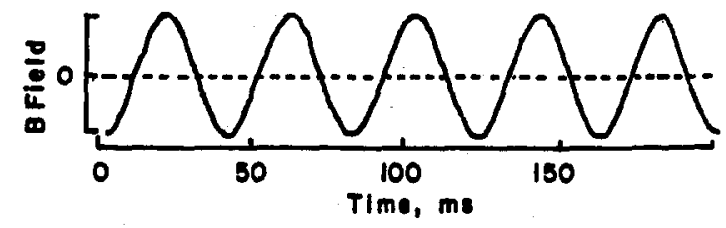

Fig. 6. Emission intensity waveforms for (a) the Ar I 415.9-nm line, (b) the Ar II 358.8-nm line, (c) the W I 400.8-nm line, (d) the continuum background and (e) the applied magnetic field. The field frequency was $25 \mathrm{~Hz}$, and the peak field amplitude was $25 \mathrm{G}$. 
intensity minima occurring during positive half cycles of the field waveform. This is more apparent for the continuum waveform.

The Ar II line and the W I line show very different behavior with only a single peak occurring during each cycle of the field waveform. For both species, the peaks occur during the positive half cycles of the field waveform. This should correspond to the current channel being at its highest vertical position.

Figures 7-9 show the effects of magnetic field strength for the Ar I and W I lines and the Ar II line, respectively. An oscillation frequency of $25 \mathrm{~Hz}$ was used in all cases. Waveforms (a) were obtained with a field strength of $25 \mathrm{G}$; while (b) and (c) were obtained with field strengths of 27 and $29 \mathrm{G}$, respectively. The magnetic field strength vs time is shown in waveform (d). The observation window in all cases was located just above the current channel in the field-free case. For the Ar I line (Fig. 7), an increase in the field strength results in a narrowing of the intensity peaks and a slight phase shift toward earlier time. The local intensity maxima also become more asymmetric with the greater intensity occurring near the positive-slope zero crossing of the magnetic field waveform.

(o)

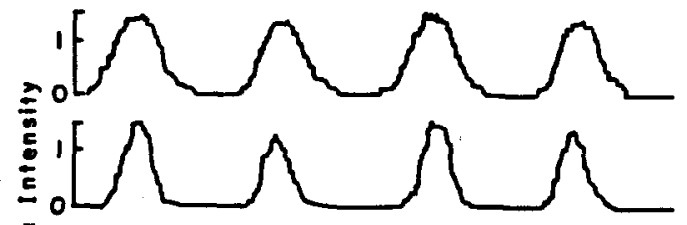

(b)

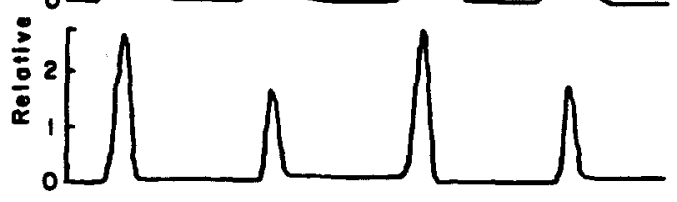

(d)

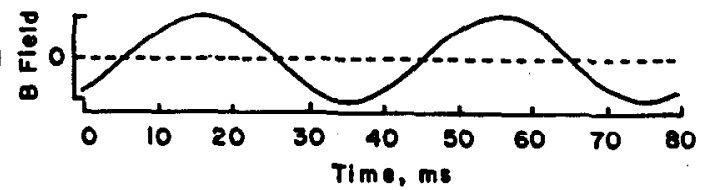

Fig. 7. Emission intensity waveforms for the Ar I 415.9-nm line using $25 \mathrm{G}$ (a), $27 \mathrm{G}$ (b) and $29 \mathrm{G}$ (c) magnetic fields. The magnetic field waveform is shown in waveform (d). The observation window was located just above the current channel for the field-free case. All other conditions are the same as for Fig. 3.

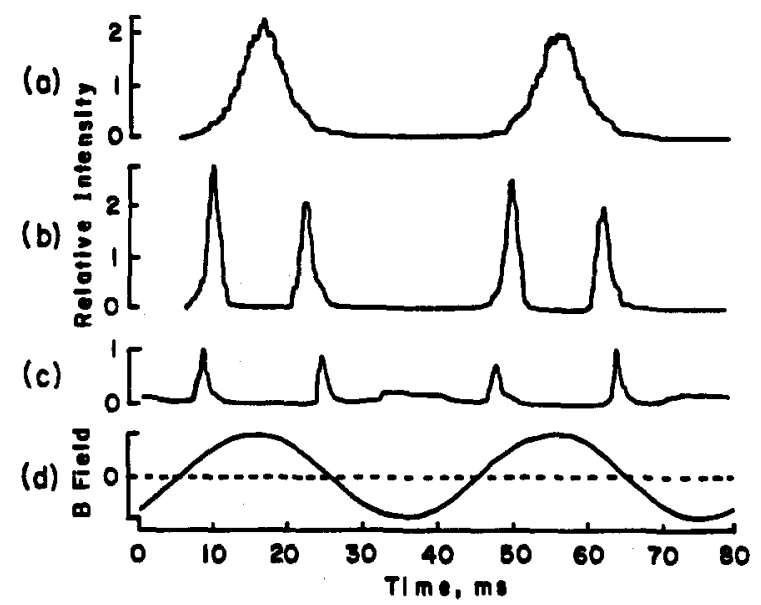

Fig. 8. Emission intensity waveforms for the W I 400.8-nm line using a $25 \mathrm{G}$ (a), a $27 \mathrm{G}$ (b) and a $29 \mathrm{G}$ (c) magnetic field. The magnetic field waveform is shown in waveform (d). All other conditions are the same as for Fig 7. 
(a)

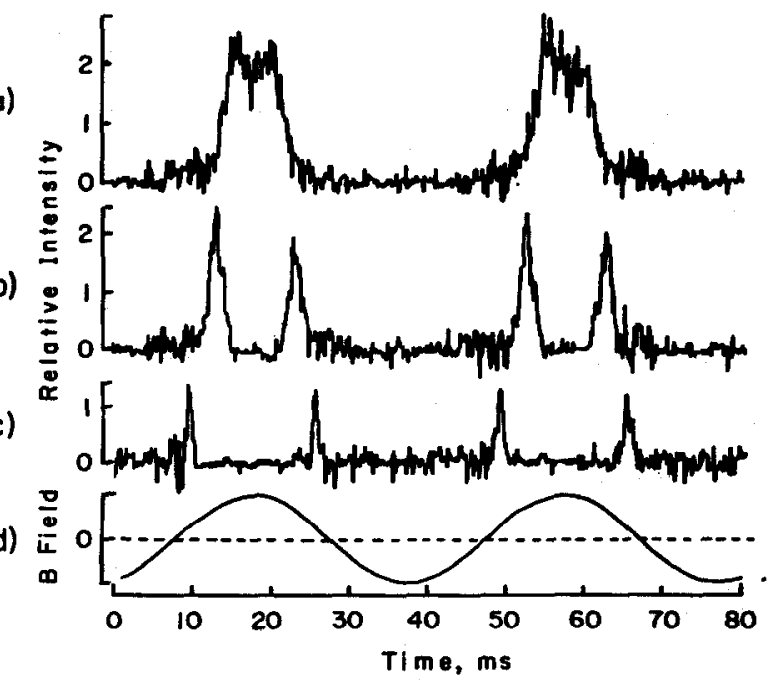

Fig. 9. Emission intensity waveforms for the Ar II $358.8-\mathrm{nm}$ line using a $25 \mathrm{G}$ (a), a $27 \mathrm{G}$ (b) and a $29 \mathrm{G}$ (c) magnetic field. The magnetic field waveform is shown in waveform (d). All other conditions are the same as for Fig. 7.

The waveforms are more complex for the $\mathrm{W}$ case (Fig. 8). As the peak field strength is increased from $25 \mathrm{G}$ to $27 \mathrm{G}$, the broad intensity peak centered near each positive peak of the field waveform appears to split into two peaks which are nearly equally spaced from the center of the single peak present at the lower field strength. For the 29-G case (c), the splitting increase and the peak amplitude decreases significantly. In addition, a new, broad, low-amplitude feature appears during each negative half cycle of the field waveform. The waveforms for the Ar II line are qualitatively similar to the $\mathrm{W}$ waveforms except that the splitting of the peaks is evident even for the $25-\mathrm{G}$ case.

Figure 10 shows a simple model of the plasma structure and motion in the $\mathbf{E} \times \mathbf{B}$ field configuration. The figure shows a cross section of the plasma in a vertical plane which bisects the arc. The dark shaded area represents the current channel. It is the only region of the plasma which contains significant amounts of Ar II. The light shaded area is a cooler mantel which emits intense Ar I radiation. Convective cooling under the current channel results in extremely steep temperature gradients in this region, and little Ar I radiation is observed. The location of the fixed observation zone is indicated by the horizontal broken lines.

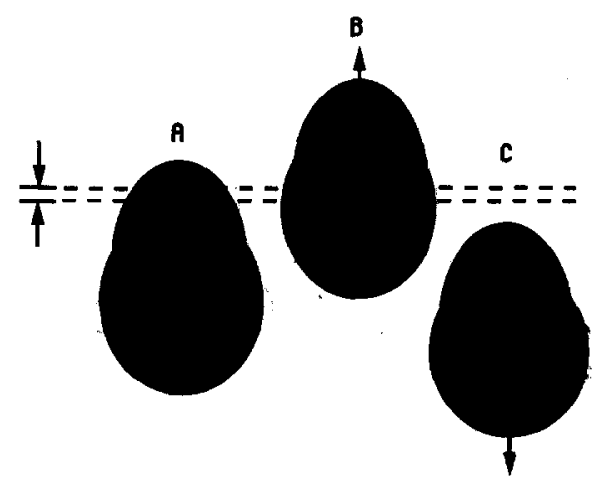

Fig. 10. Simplified model of the movement of the plasma in the external magnetic field. The plasma electric field is normal to the figure and the applied magnetic field is parallel to the left-right axis. The horizontal lines indicate the position of the observation window. $A$, no magnetic field (zero-crossings of the field waveform); B, positive excursions of the magnetic field waveform, $C$, negative excursions of the field waveform. 
For case A, no magnetic field is present, and the current channel is in its quiescent location. Here the mantel region is being viewed. Cases $B$ and $C$ show the peak displacements of the current channel caused by a low-amplitude oscillating field comparable to that used for waveforms (a) of Figs 7-9. Note that for the Ar I waveforms in Fig. 7, the greatest intensity occurs at the zero-crossings of the magnetic field waveform. This corresponds to the field-free case A in Fig. 10. During each positive half cycle of the magnetic field waveform, the current channel moves into the observation zone (case B). The mantel has moved out of the observation zone, and little Ar I radiation is detected. During negative half cycles of the field waveform, the current channel moves downward, the mantel moves out of the observation zone, and relatively little radiation is observed from either Ar II or Ar I species.

For larger peak-field amplitude, the current channel moves through and beyond the observation zone during positive half cycles of the magnetic field waveform. This causes the single peak observed for the W I line and the Ar II line to split into two narrow peaks, one as the channel moves through the observation zone during increasing field strength, and one as the channel moves back through the observation zone during decreasing field strength. As the peak field amplitude increases further, the emitting region passes through the observation zone more rapidly, and the emission peaks become narrower. This is clearly seen in Figs 7-9.

Figures 11 and 12 show the effect of field frequency on the Ar II line waveform and the $\mathrm{Cu} I$ line waveform, respectively. The peak magnetic field strength was $25 \mathrm{G}$ for all cases. Waveforms (a) were obtained with an oscillation frequency of $100 \mathrm{~Hz}$; while (b), (c), (d) and (e) were obtained at frequencies of $200,300,400$ and $500 \mathrm{~Hz}$, respectively. The plots labeled (f) are for the magnetic field waveform. In all cases, the observation zone was located at the center of the current channel for the field-

(a)

(b)

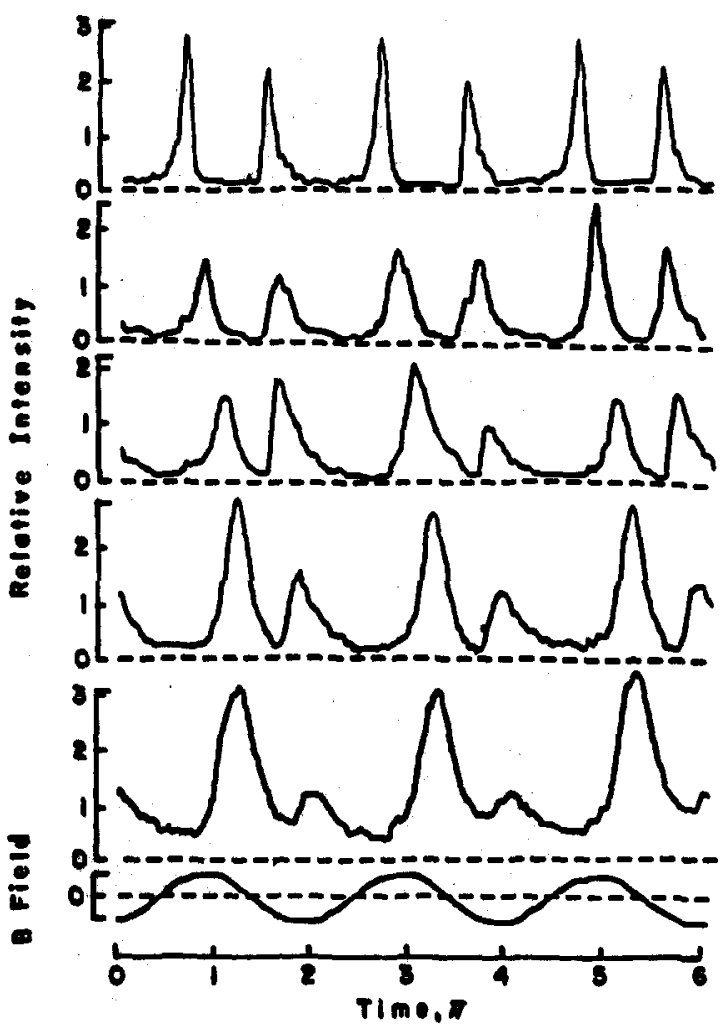

Fig. 11. Emission intensity waveforms for the Ar II 358.8-nm line using magnetic field frequencies of (a) $10 \mathrm{~Hz}$, (b) $200 \mathrm{~Hz}$, (c) $300 \mathrm{~Hz}$, (d) $400 \mathrm{~Hz}$, and (e) $500 \mathrm{~Hz}$. Peak field amplitude was $25 \mathrm{G}$ in all cases. Waveform (f) shows the magnetic field. For all cases, the observation window was located at the center of the current channel for the field-free case. 
(o)

(b)

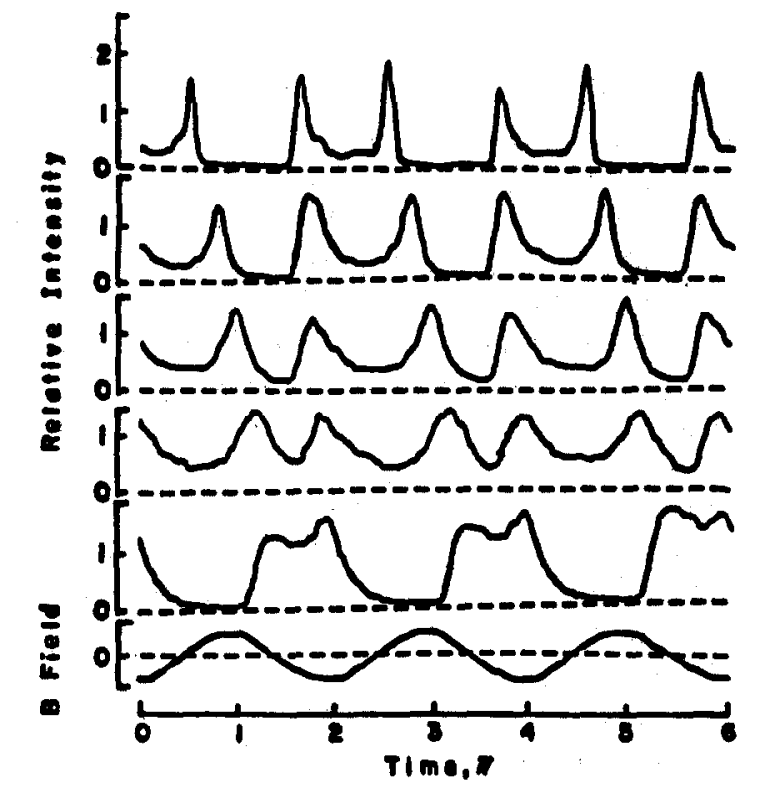

Fig. 12. Emission intensity waveforms for the $\mathrm{Cu}$ I $324.7-\mathrm{nm}$ line using magnetic field frequencies of (a) $100 \mathrm{~Hz}$, (b) $200 \mathrm{~Hz}$, (c) $300 \mathrm{~Hz}$, (d) $400 \mathrm{~Hz}$, and (e) $500 \mathrm{~Hz}$. Peak field amplitude was $25 \mathrm{G}$ in all case. Waveform (f) shows the magnetic field. For all cases, the observation window was located at the center of the current channel for the field-free case.

free case. The Ar I line as well as all lines investigated from added analyte species produced waveforms which are qualitatively similar to the $\mathrm{Cu}$ waveforms in Fig. 12 .

For the $100-\mathrm{Hz}$ case, both the $\mathrm{Ar}$ II and the $\mathrm{Cu}$ I waveforms are qualitatively similar to the 25- $\mathrm{Hz}$ Ar II waveform at slightly lower magnetic field strength (see Fig. 9b). The first peak corresponds to the plasma moving upward through the observation zone; while the second peak corresponds to the plasma moving downward through the observation zone. The leading edge of the first peak and the falling edge of the second peak are more diffuse because of the smaller thermal gradients near the top edge of the current channel. During the interval between the first and second peaks, the region under the current channel is in the observation zone and relatively little emission is seen from either species. During the interval between the second and third peaks, the tail-flame above the current channel is in the observation zone, and there is significant $\mathrm{Cu} \mathrm{I}$ emission. The steep falling edge of the first peak and rising edge of the second peak correspond to the lower plasma boundary passing through the observation window. Convection results in this boundary being much sharper.

As the frequency is increased, the first peak (and all odd number peaks) becomes broader and shifts to later time. The second peak (and all even number peaks) also broadens significantly, but the shift to later time is smaller, particularly for the $\mathrm{Cu} I$ line. At $500 \mathrm{~Hz}$, the two peaks for $\mathrm{Cu}$ in each magnetic field cycle begin to coalesce.

These waveforms suggest that at the higher frequencies, the structure of the current channel changes significantly. Previous studies using a $\mathrm{cw}$ magnetic field to induce rotation of the current channel in a concentric-electrode DCP indicated that for rotation frequencies greater than a few hundred $\mathrm{Hz}$, the current channel loses its discrete nature and becomes delocalized in the form of a dome covering the ring-shaped anode. With increasing frequency, the Ar II radiation showed less periodic character. Some delocalization of the Ar II radiation also is suggested by Fig. 11. As the oscillation period approaches the gas thermal relaxation time, the dc component of the radiation passing through the fixed observation window should increase. The phase relationships in Figs 11 and 12 are more difficult to understand. The delocalization of the plasma may be accompanied by other drift motions caused by the interaction of the magnetic field with the time-varying components of the plasma electric field [35]. 


\section{Conclusions}

The experimental system used for the work reported here has several advantages over previously described systems used in this laboratory $[1,2]$. First, the use of an adjustable interelectrode angle has allowed the selection of conditions which result in much greater plasma stability and lower noise. This results in greater signal-to-noise ratios. Greater positional stability of the plasma also has resulted in improved spatial resolution. Second, the use of an adjustable-frequency magnet power supply has allowed a more detailed study of inertial effects in the magnetic-field-induced movement of the arc current channel. Third, by observing the plasma through a hole in one of the pole pieces, it was not necesssary to use a bent magnetic field axis as was the case in previous studies. Finally, the new system allows more accurate measurement of the phase shifts between the applied magnetic field and the emission waveforms.

The current channel of the two-electrode DCP is very susceptible to magnetic-field manipulation. Magnetic field strengths in the range from a few $G$ to about $30 \mathrm{G}$ seem most appropriate. Weaker fields produce only small displacements of the current channel from its field-free location. Magnetic fields (cw) greater than about $30 \mathrm{G}$ lead to plasma instabilities and turbulent gas flows.

The change in shape of the current channel produced by a cw magnetic field can be used to improve sample-plasma interaction for the case where the $\mathbf{E} \times \mathbf{B}$ drift motion of plasma electrons is directed in the positive vertical direction. This appears to form an enlarged pocket under the current channel. Increased residence time of the sample aerosol in this region of the plasma may explain the greater emission intensities observed there.

There is strong indication of current-channel delocalization and significant changes in plasma structure for oscillating magnetic fields with frequencies greater than about $100 \mathrm{~Hz}$. Operation with frequencies greater than $500 \mathrm{~Hz}$ would be most interesting but will require a more robust current source. The use of an oscillating magnetic field may result in a physically larger and more diffuse plasma. This may result in a reduced thermal barrier under the plasma in the region of sample aerosol penetration. A similar delocalization of the arc current channel recently has been reported for a concentricelectrode arc device in which a magnetic field parallel to the electrode axis causes the arc current channel to rotate around the edge of a cylindrical graphite anode [4,5].

\section{REFERENCES}

[1] K. Trivedi, S. Tanguay, M. Matties and R. Sacks, Appl. Spectrosc. 41, 833 (1987).

[2] K. Trivedi, D. Coll and R. Sacks, Appl. Spectrosc. 42, 1025 (1988).

[3] B. T. Buckley, C. B. Boss and J. S. Gentry, FACSS XIV, Detroit, Michigan paper No. 184 (1987).

[4] D. Slinkman and R. Sacks, Appl. Spectrosc. 44, 76 (1990).

[5] D. Slinkman and R. Sacks, Appl. Spectrosc. 44, 83 (1990).

[6] N. Krasnobaeva, Ju. Charizanov and Z. Zadgorska, Spectrochim. Acta 24B, 473 (1969).

[7] Yu. Harizanov and Z. Zadgorska, Spectrochim. Acta 25B, 29 (1969).

[8] M. Todorvic, V. Vukanović and V. Georgijivić, Spectrochim. Acta 24B, 571 (1969).

[9] V. Vukanovic, V. Georgijivić and M. Todorović, Spectrochim. Acta 24B, 555 (1969).

[10] S. Belchev and P. Georgieva, Spectrochim. Acta 35B, 367 (1974).

[11] D. Lummerzheim and H. Nickel, Z. Anal. Chem. 245, 267 (1969).

[12] D. Leushacke, D. Lummerzheim and H. Nickel. Z. Anal. Chem. 250, 225 (1970).

[13] M. Yamamoto, Japan J. Appl. Phys. 1, 235 (1962).

[14] S. Tanguay and R. Sacks, Appl. Spectrosc. 43, 918 (1989).

[15] S. Tanguay and R. Sacks, Appl. Spectrosc. 42, 576 (1988).

[16] L. McCaig and R. Sacks, Appl. Spectrosc. 43, 912 (1989).

[17] L. McCaig and R. Sacks, Appl. Spectrosc. 44, (submitted).

[18] R. Simonneau and R. Sacks, Appl. Spectrosc. 43, 141 (1989).

[19] R. A. Kruger, R. M. Bombelka and K. Laqua, Spectrochim. Acta, 35B, 581 (1980).

[20] C. E. Schmidt and R. D. Sacks, Spectrochim. Acta 38B, 557 (1983).

[21] R. J. Decker, Spectrochim. Acta 35B, 19 (1980).

[22] E. Kranz, Emissionssektroskopie, p. 160, Akademie, Berlin (1964).

[23] J. A. Holcombe, R. H. Eklund and K. E. Grive, Anal. Chem. 50, 2097 (1978).

[24] N. A. Fuchs, The Mechanisms of Aerosols. Pergamon Press, New York (1966). 
[25] G. H. Lee, J. P. Shields and E. H. Piepmeier, Spectrochim. Acta 43B, 1273 (1988).

[26] G. A. Meyer, Spectrochim. Acta 42B, 333 (1987).

[27] L. Y. Hara and M. L. Parsons, Anal. Chem. 57, 841 (1985).

[28] T. R. Mattoon and E. H. Piepmeier, Anal. Chem. 55, 1045 (1983).

[29] S. F. Valente and W. G. Schrenk, Appl. Spectrosc. 24, 197 (1970).

[30] W. E. Rippetoe and T. J. Vickers, Anal. Chem. 47, 2082 (1975).

[31] J. P. Shields, G. H. Lee and E. H. Piepmeier, Appl. Spectrosc. 42, 684 (1988).

[32] D. A. Murdick, Jr and E. H. Piepmeier, Anal. Chem. 46, 678 (1974).

[33] L. R. Layman and F. E. Lichte, Anal. Chem. 54, 638 (1982).

[34] E. Michaud-Poussel and J. M. Mermet, Spectrochim. Acta 41B, 49 (1986).

[35] F. F. Chen, Introduction to Plasma Physics. Plenum Press, New York (1974). 